\title{
Width Optimization of Array Electrode for Human Lung Electrical Resistance Tomography System Based on prior Knowledge
}

\author{
Liqing Xiao $\mathbb{D}$ \\ School of Mechanical and Electrical Engineering, Huainan Normal University, Huainan 232038, China \\ Correspondence should be addressed to Liqing Xiao; lqx1981@tju.edu.cn
}

Received 8 April 2021; Revised 23 April 2021; Accepted 3 May 2021; Published 11 May 2021

Academic Editor: Ahmed Mostafa Khalil

Copyright (c) 2021 Liqing Xiao. This is an open access article distributed under the Creative Commons Attribution License, which permits unrestricted use, distribution, and reproduction in any medium, provided the original work is properly cited.

In electrical resistance tomography (ERT) technology for human lung, under the same experimental conditions, the width of the sensitive field boundary electrode has a significant impact on the calculation accuracy of the inverse problem besides the finite element model (FEM) topology. Aiming to improve the quality of reconstructed images, the FEM for human lung was set up based on prior knowledge. On this basis, the electrode width of the FEM was optimised by comparing the morbidity degrees of the sensitivity matrix and Hessian matrix, the uniformity of sensitivity distribution, and the quality of reconstructed images, which can improve the accuracy of solving the inverse problem significantly.

\section{Introduction}

In recent years, critical respiratory diseases have become a hot area of critical care medicine and one of the primary directions of disease prevention and control in China with features of high complexity, morbidity, mortality, and risk of great harm. Respiratory diseases have become one of the diseases that seriously threaten the health of Chinese residents, according to the statistical results of Vols. 2013-2017 of China Health and Family Planning Statistical Yearbook and Vols. 2018 and 2019 of China Health Statistics Yearbook [1-7]. Increasing population ageing, national smog and haze, influenza pneumonia, highly pathogenic avian influenza, and other factors will increase the morbidity and mortality of critical respiratory diseases in China, which has caused widespread attention. Compared with the epidemiological results of foreign countries, the status quo of the treatment of critical respiratory diseases in China is not optimistic. The mortality rate of critical respiratory diseases in China is much higher than that in Western developed countries. Therefore, the prevention and treatment of critical respiratory diseases are acute.

Due to the complexity of critical respiratory diseases, the current clinical treatment plan depends on the individual patient. Therefore, timely assessing the effectiveness of medical measures and accurately grasping the changes in the progress of lung diseases is an essential basis for successful treatment of critical respiratory diseases. However, currently, there is a lack of clinically effective means for real-time monitoring and quantitative evaluation of critical respiratory diseases.

Electrical tomography (ET) technology consists of 4 different branches, namely, electrical impedance tomography (EIT) [8-14], ERT [15-20], electrical capacitance tomography (ECT) [21-27], and electromagnetic tomography (EMT) [28-33], among which the ERT technology is a new generation of medical imaging technology and a simplified form of the EIT technology when only the change of conductivity/resistivity of the sensitive field is considered, having three outstanding advantages of functional imaging, no damage, and medical image monitoring. Compared with other monitoring methods, ERT technology has incomparable advantages in real-time monitoring and quantitative evaluation of critical respiratory diseases.

\section{Basic Principles of Human Lung ERT}

Human lung ERT technology is based on the characteristics that different human tissues and organs have different conductivities/resistivities. Various physiological and pathological conditions often correspond to conductivity/resistivity changes of 
certain tissues and organs. Firstly, a safe excitation current signal is applied on the electrode array on the body surface to establish a sensitive field in the lungs. Secondly, the effective boundary voltage of the sensitive field is obtained through the data acquisition circuit. Finally, according to the effective boundary voltage of the sensitive field, the image reconstruction algorithm is employed to solve the inverse problem of the human lung ERT to obtain the conductivity/resistivity image distribution of tissues and organs (including lungs, heart, and spine) in the sensitive field.

The current density J, electrical conductivity distribution $\sigma$, electrical potential distribution $\Phi$, and electric field strength $\mathbf{E}$ in the sensitive field region $\Omega$ of the human lung ERT satisfy Equations (1)-(3). By simplifying these equationuations, the mathematical model of the sensitive field of the human lung ERT can be obtained, as shown in Equation (4):

$$
\begin{aligned}
\nabla \cdot \mathbf{J} & =0, \\
\mathbf{J} & =\sigma \cdot E, \\
\mathbf{E} & =-\nabla \Phi, \\
\nabla \cdot(\boldsymbol{\sigma} \nabla \boldsymbol{\Phi}) & =0 .
\end{aligned}
$$

The calculation of forward problem for human lung ERT is to calculate the potential distribution of the sensitive field generated by the given boundary stimulating electrical signal to obtain the effective boundary voltage value for solving the inverse problem, based on the known or given conductivity/ resistivity distributions of tissues and organs (including the lungs, heart, and spine) within the sensitive field region. Due to the irregular geometry of the measured field, it is a challenge to use analytical methods to obtain analytical solutions to the positive problem through theoretical derivation. The numerical calculation method of FEM, which is more adaptable to the geometric shape of the measured field, is usually used to calculate the forward problem.

In the FEM-based calculation process of the forward problem of the human lung ERT, the current density J, electrical conductivity distribution $\sigma$, electrical potential distribution $\Phi$, and total coefficient matrix $\mathbf{K}$ in the region $\Omega$ of the sensitive field satisfy the following relationship, as shown in equations (5)-(8):

$$
F(\Phi)=\frac{1}{2} \int_{\Omega} \nabla \cdot(\boldsymbol{\sigma} \nabla \boldsymbol{\Phi}) \mathrm{d} \Omega-\oint_{S} \mathbf{J} \Phi \mathrm{d} S=\min ,
$$

where $S$ is the boundary of the region $\Omega$ of the sensitive field:

$$
\begin{aligned}
F(\boldsymbol{\Phi}) & =\frac{1}{2} \boldsymbol{\Phi}^{T} K \boldsymbol{\Phi}-\boldsymbol{\Phi}^{T} \mathbf{J}, \\
\mathbf{K} & =\sum_{e} \widetilde{\mathbf{K}}_{e} \\
{[\mathbf{K}][\boldsymbol{\Phi}] } & =[\mathbf{J}] .
\end{aligned}
$$

The so-called image reconstruction is to solve the conductivity/resistance image distribution of tissues and organs in the sensitive field based on the given boundary excitation electrical signal and the corresponding effective boundary voltage, which is essential to the human lung ERT. In the two types of different image reconstruction methods for the human lung ERT, although dynamic imaging can achieve real-time imaging, static imaging has a wider application range and higher clinical value because the latter can present the absolute value of the conductivity of tissues and organs. People can diagnose different tissues and organs (including lungs, heart, and spine) as normal tissues or diseased ones according to their conductivity/resistivity values.

\section{Comparison of Different Electrode Widths of Sensitive Fields}

When the boundary curve equationuation of lungs is determined, the width of the sensitive field boundary electrode only depends on the electrode angle. Although the optimisation of electrode width of the ERT system is realized in [34], only four different electrode widths were compared, and there were some irrationalities in comparing the calculation accuracy of forward problem and selecting the image reconstruction results of Newton-Raphson algorithm. In the process of image reconstruction for the Newton-Raphson algorithm, the sensitivity matrix can be fixed, so it is meaningful to compare the sensitivity matrix. However, the accuracy of forward problem cannot be evaluated by only one set of distribution, and the optimal image reconstruction result cannot be determined in advance according to the image correlation coefficient, but only according to the algorithm error.

Now, the FEM for ERT is chosen and shown in Figure 1, which contains 537 nodes and 880 finite elements and the number of electrodes is 16 . The data acquisition adopts the adjacent incentive mode, which is widely used in ERT. The excitation current is $1 \mathrm{~mA}$. The number of elements used in the FEM has a certain influence on the simulation accuracy, and the number is determined by comparing the root mean square values, the time of calculating the effective boundary voltage values, and the time of calculating the sensitivity matrix. The FEM not only improves the degree of region fitting but also improves the ill-conditioned degree of the sensitivity matrix [34]. Based on prior knowledge, the internal tissue and organ distribution of the FEM for human lung ERT is set up, as shown in Figure 2. On this basis, the morbidity degrees of sensitivity matrix and Hessian matrix, the uniformity of sensitivity distribution, and the quality of reconstructed images at electrode angles of 1.8750 degrees, 3.7500 degrees, 5.6250 degrees, 7.5000 degrees, 9.3750 degrees, 11.2500 degrees, and 13.1250 degrees are compared and analysed.

3.1. Comparison of Ill-Conditioned Degrees of Sensitivity Matrix and Hessian Matrix. The internal tissue and organ distribution in the FEM for human lung ERT is shown in Figure 2, and the condition numbers of the sensitivity matrix are $5.3077 \times 10^{6}, 5.2601 \times 10^{6}, 5.0142 \times 10^{6}, 4.8788 \times 10^{6}$, $4.5361 \times 10^{6}, 4.3041 \times 10^{6}$, and $3.8943 \times 10^{6}$ at electrode 


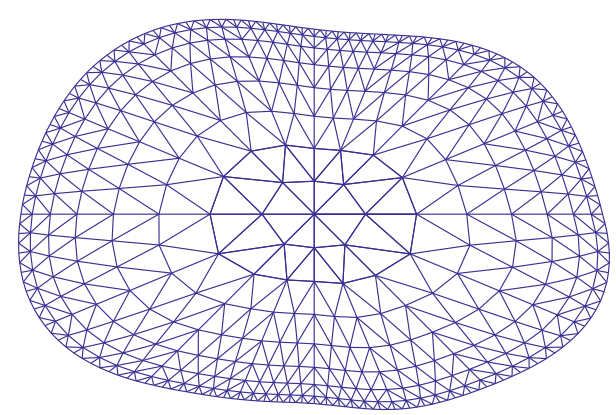

Figure 1: Schematic diagram of the FEM for ERT.

angles of 1.8750 degrees, 3.7500 degrees, 5.6250 degrees, 7.5000 degrees, 9.3750 degrees, 11.2500 degrees, and 13.1250 degrees, respectively. When the regularization factor is $10^{-5}$, the condition numbers of Hessian matrix are $1.8154 \times 10^{2}$, $1.7930 \times 10^{2}, \quad 1.8055 \times 10^{2}, \quad 1.7888 \times 10^{2}, \quad 1.7993 \times 10^{2}$, $1.7838 \times 10^{2}$, and $1.7928 \times 10^{2}$ at electrode angles of 1.8750 degrees, 3.7500 degrees, 5.6250 degrees, 7.5000 degrees, 9.3750 degrees, 11.2500 degrees, and 13.1250 degrees, respectively.

\subsection{Comparison of Uniformity of Sensitivity Distribution.}

The electrodes are evenly distributed and numbered anticlockwise. The electrode pair N-M means that the electrodes $\mathrm{N}$ and $\mathrm{M}$ are used as excitation electrodes or measurement electrodes. When the electrode pair 1-2 is the excitation electrode, parts of the sensitivity distribution corresponding to the FEM are shown in Figure 3, and the maximum values, the minimum values of the corresponding sensitivity matrices, and the calculated $P$ values by using Equation (9) are shown in Table 1, which are used to evaluate the uniformity of sensitivity distribution. It is usually believed that the accuracy of inverse problem can be improved by improving the uniformity of sensitivity distribution.

$$
P=\frac{\sum_{i=1} \sum_{j=2}^{M}\left|p_{\mathrm{ij}}\right|}{M},
$$

where $p_{i j}$ is given by

$$
p_{\mathrm{ij}}=\frac{S_{i j}^{\mathrm{dev}}}{S_{\mathrm{ij}}^{\mathrm{avg}}}
$$

where $S_{\mathrm{ij}}$ is the sensitivity of the electrode pair of $i-j$ and $S_{\mathrm{ij}}^{\mathrm{avg}}$ and $S_{\mathrm{ij}}^{\mathrm{dev}}$ are the mean and standard deviation of the sensitivity matrix with the triangular finite element area coefficient.

It can be seen from Table 1 and Figure 3 that the sensitivity distribution is the most uniform when the electrode angle is 13.1250 degrees.

3.3. Comparison of Image Reconstruction Quality. Based on prior knowledge, 6 distributions of internal tissues and organs in the FEM for human lung ERT are shown in Figure 4 . The dark red area is the pathological lung tissue. The finite element model shown in Figure 1 is refined and used to calculate the forward problem and obtain the boundary voltage measurement values. The image reconstruction algorithm draws on the modified Newton-Raphson algorithm proposed in [35], and the specific process is as follows:

Step 1: set the algorithm parameters, including the maximum iteration number and the regularization factor, which have an important influence on the results of image reconstruction.

Step 2: calculate the sensitivity matrix $f^{\prime}\left(\rho^{(0)}\right)$ corresponding to the internal tissue and organ distribution of the FEM for human lung ERT in Figure 2.

Step 3: randomly generate the diagonal matrix $\mathbf{M}_{\text {numexnume }}^{(k)}$ the initial seed group with the particle

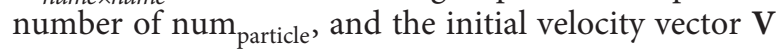
of the particles and optimise the main diagonal elements of the diagonal matrix $\mathbf{M}_{\text {numexnume }}^{(k)}$ by using the balance method based on the improved particle group algorithm. The fitness function selected is presented as

$$
F\left(\mathbf{M}_{\text {numeхnume }}^{(k)}\right)=\frac{P}{\operatorname{cond}(\mathbf{Y})},
$$

where cond is the condition number, the expression of $\mathbf{Y}$ is as shown in Equation (12), and $P$ is a penalty function, the expression is as shown in Equation (13):

$$
Y=\mathbf{M}_{\text {numeхnume }}^{(k)} \cdot\left\{\left[f^{\prime}\left(\rho^{(0)}\right)\right]^{T} f^{\prime}\left(\rho^{(0)}\right)+\mu^{(k)} E\right\}^{-1} \cdot\left[f^{\prime}\left(\rho^{(0)}\right)\right]^{T},
$$

$$
P=\left\{\begin{array}{l}
1 \quad \operatorname{cond}\left(\mathbf{M}_{\text {numexnume }}^{(k)}\right) \leq \operatorname{cond}(\mathbf{Y}) \\
10^{-10} \quad \operatorname{cond}\left(\mathbf{M}_{\text {numexnume }}^{(k)}\right)>\operatorname{cond}(\mathbf{Y})
\end{array}\right.
$$

Step 4: Ttke the reconstruction result of the linear backprojection algorithm as the initial estimated value $\rho^{(1)}$ of the resistivity distribution, calculate the forward problem to obtain the calculated value $v^{(1)}$ of the boundary voltage of the sensitive field, and substitute it into Equation (14) to get $E^{(1)}$, and substitute $\rho^{(1)}$ and $E^{(1)}$ into the optimal value $\mathbf{b}_{\text {estr }}$ and the minimum error value $b_{\text {est } f}$ of the resistivity distribution of the sensitive field: 


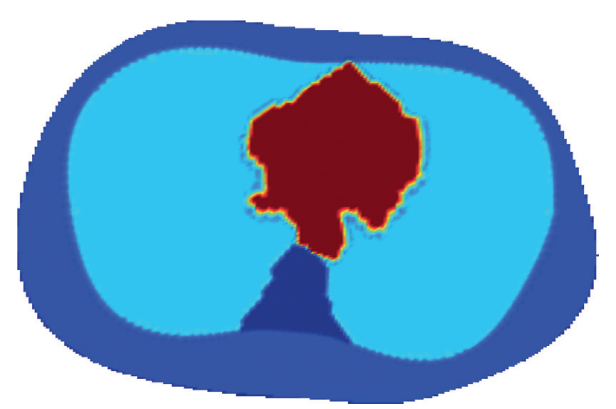

FIgURE 2: Schematic diagram of the internal tissue and organ distribution based on prior knowledge.

$$
E^{(k)}=\frac{1}{2}\left(\left\|v^{(k)}-v_{0}\right\|_{2}\right)^{2},
$$

where $v_{0}$ is the measured value of the boundary voltage of the sensitive field.

Step 5: the termination of the algorithm is based on the criteria of the number of iterations and the allowable error of the algorithm. If it is satisfied, the algorithm returns the optimal value $\mathbf{b}_{\text {estr }}$ and the minimum error value $b_{\text {est } f}$ of the resistivity distribution of the sensitive field; otherwise, go to Step 6.

Step 6: calculate $\Delta \rho^{(k+1)}$ according to equations (15) and (16):

$$
\begin{aligned}
& \Delta \rho_{1}^{(k+1)}=-Y\left(f\left(\rho^{(k)}\right)-\mathbf{v}_{0}\right) . \\
& \Delta \rho^{(k+1)}=\left(\mathbf{M}_{\text {nume } \times \text { nume }}^{(k)}\right)^{-1} \cdot \Delta \rho_{1}^{(k+1)} .
\end{aligned}
$$

Step 7: correct the resistivity distribution $\rho^{(k+1)}$ of the sensitive field according to equation (17), calculate the forward problem to obtain the calculated value $\mathbf{v}^{(k+1)}$ of the boundary voltage of the sensitive field, and substitute it into equation (14) to obtain $E^{(k+1)}$. If $E^{(k+1)}<b_{\text {est } f}$, replace the minimum error value $b_{\text {est } f}$ of the algorithm and the optimal value $\mathbf{b}_{\text {estr }}$ of the resistivity distribution of the sensitive field with $E^{(k+1)}$ and $\rho^{(k+1)}$, respectively, and return to Step 5; otherwise, return to Step 5 directly:

$$
\rho^{(k+1)}=\rho^{(k)}+\Delta \rho^{(k+1)}
$$

Using the abovementioned modified Newton-Raphson image reconstruction algorithm, the reconstructed images are shown in Figure 5 using a PC with a CPU of Intel (R) Core(TM) i7-4510U $2.60 \mathrm{GHz}$ and $8 \mathrm{~GB}$ RAM.

And, the image correlation coefficient, image relative error $e$, and absolute error $A E$ are shown in Tables 2-4, and their expressions are shown in Equations (18)-(20), respectively.

$$
\begin{aligned}
& \rho=\frac{\sum_{i=1}^{L}\left(\widehat{\mathbf{g}}_{i}-\overline{\widehat{g}}\right) \cdot\left(\mathbf{g}_{i}-\bar{g}\right)}{\sqrt{\sum_{i=1}^{L}\left(\widehat{\mathbf{g}}_{i}-\overline{\widehat{g}}\right)^{2} \sum_{i=1}^{L}\left(\mathbf{g}_{i}-\bar{g}\right)^{2}}}, \\
& e=\frac{\|\mathbf{g}-\widehat{\mathbf{g}}\|_{2}}{\|\mathbf{g}\|_{2}} \times 100 \%,
\end{aligned}
$$

where $\mathbf{g}$ and $\widehat{\mathbf{g}}$ are the setting value of electrical conductivity distributions of tissues and organs in the FEM of the human lung ERT and corresponding calculated value by solving the inverse problem with the modified Newton-Raphson algorithm, respectively, and their averaged values are $\bar{g}$ and $\overline{\hat{g}}$, respectively, $L$ is the number of triangular finite elements for dividing the sensitive field of the ERT, and $i$ is the finite element number:

$$
\mathrm{AE}=\frac{1}{5} \sum_{i=1}^{5}\left|\frac{\rho_{i}-\widehat{\rho}_{i}}{\rho_{i}}\right|,
$$

where $\rho_{i}$ and $\hat{\rho}_{i}(i=1,2,3,4,5)$ are conductivity values of the tissues and organs (including the heart, spine, fat, and normal and pathological lung tissue) in the FEM of the human lung ERT and that obtained by solving the inverse problem with the modified Newton-Raphson algorithm.

It can be seen from Tables $2-4$ that, at electrode angles of 1.8750 degrees, 3.7500 degrees, 5.6250 degrees, 7.5000 degrees, 9.3750 degrees, 11.2500 degrees, and 13.1250 degrees, respectively, the average values of image correlation coefficients are $0.8691,0.8467,0.8567,0.8259,0.8388,0.8162$, and 0.7744 , the average values of image relative error are $40.8608 \%, 44.1065 \%, 42.5599 \%, 44.2302 \%, 42.5519 \%$, $44.6313 \%$, and $49.4449 \%$, and the average values of absolute errors are $0.1112,0.1180,0.1030,0.1500,0.1386,0.1518$, and 0.1551 . According to Figure 5, the quality of reconstructed images is the highest when the electrode angle is 1.8750 degrees.

Base on the comprehensive comparison in the aspect of the morbidity degrees of the sensitivity matrix and Hessian matrix, the uniformity of sensitivity distribution, and the quality of reconstructed images at electrode angles of 1.8750 degrees, 3.7500 degrees, 5.6250 degrees, 7.5000 degrees, 9.3750 degrees, 11.2500 degrees, and 13.1250 degrees, the optimised result of the electrode angle is 1.8750 degrees. 

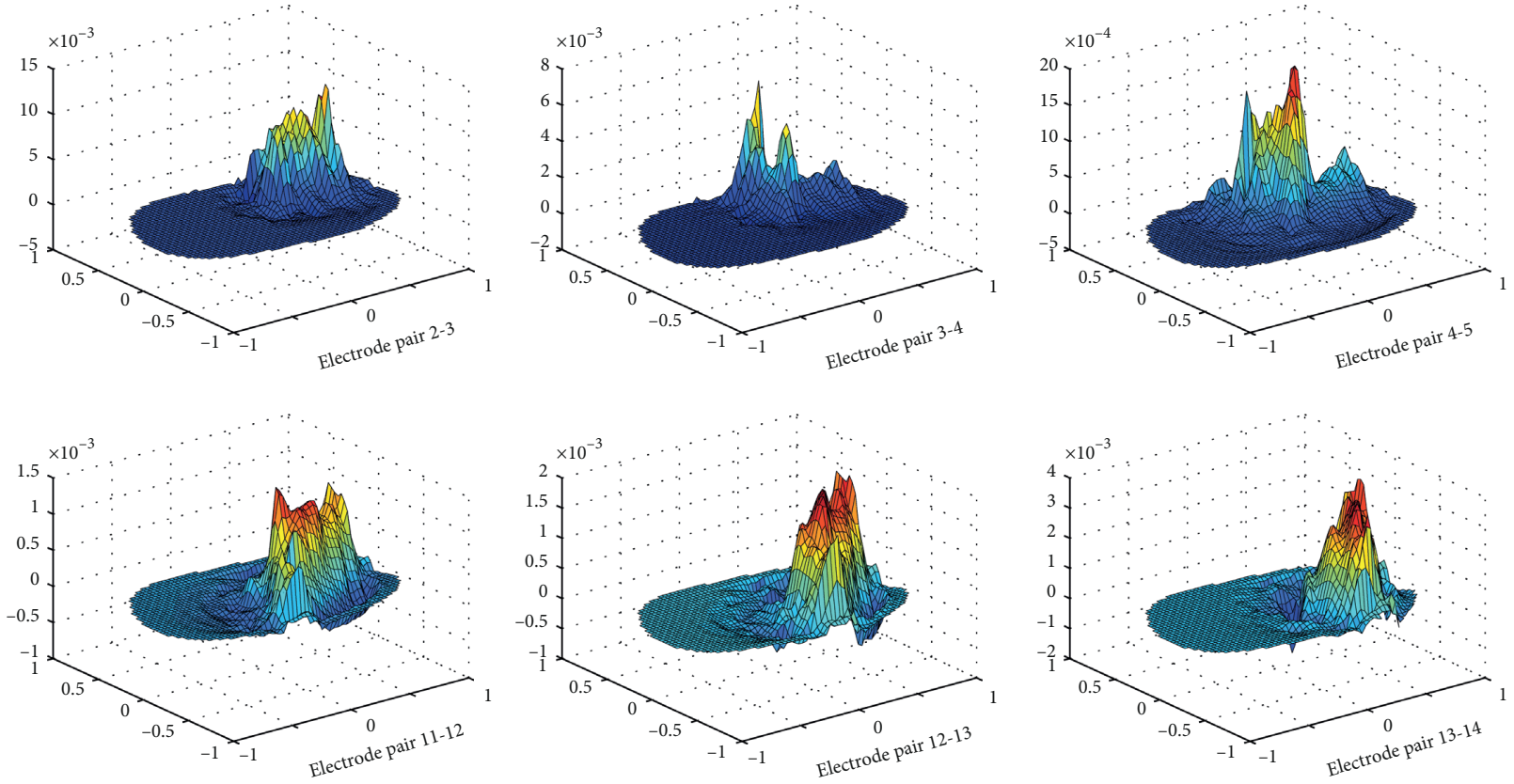

(a)
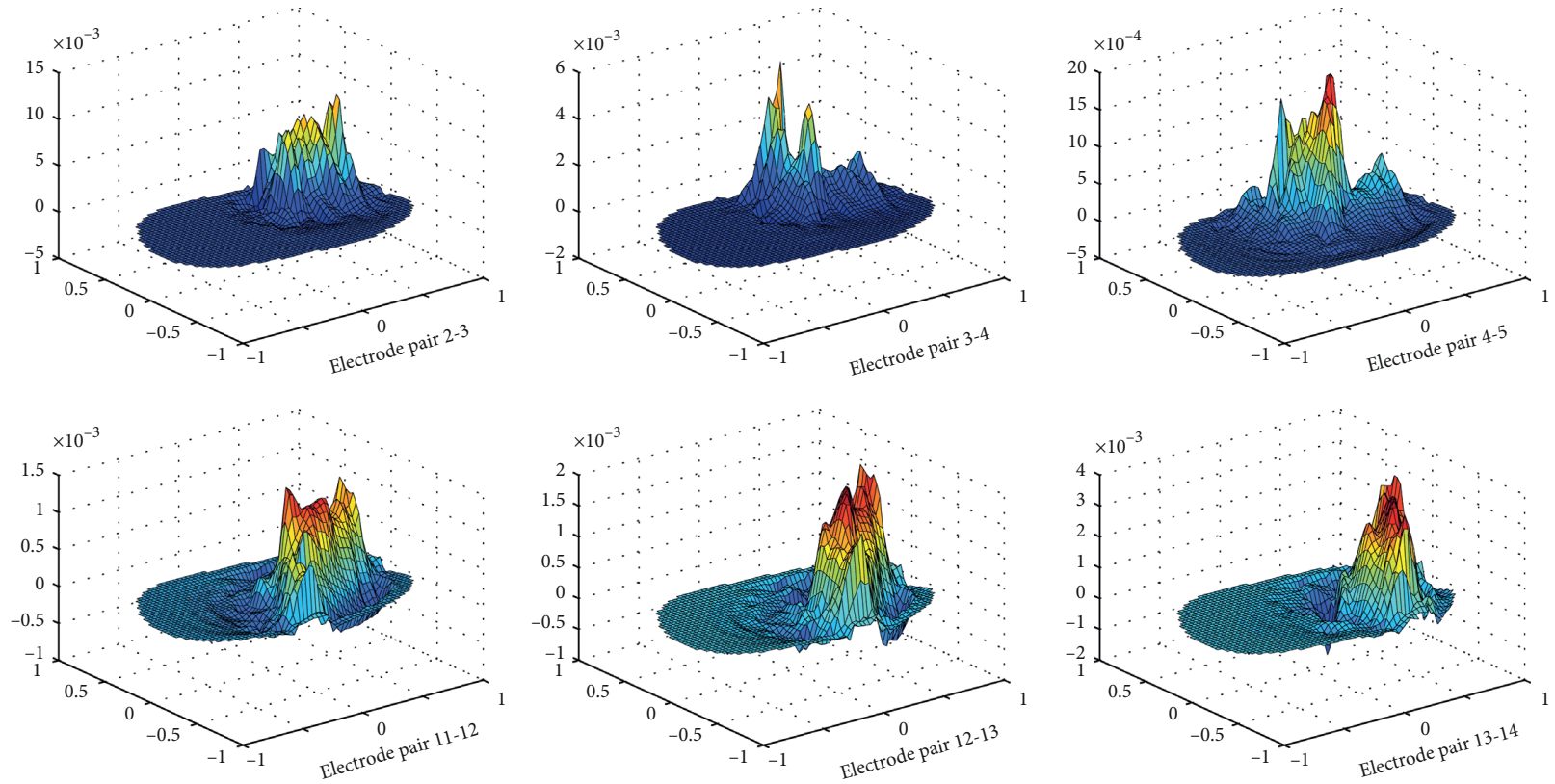

(b)

Figure 3: Continued. 

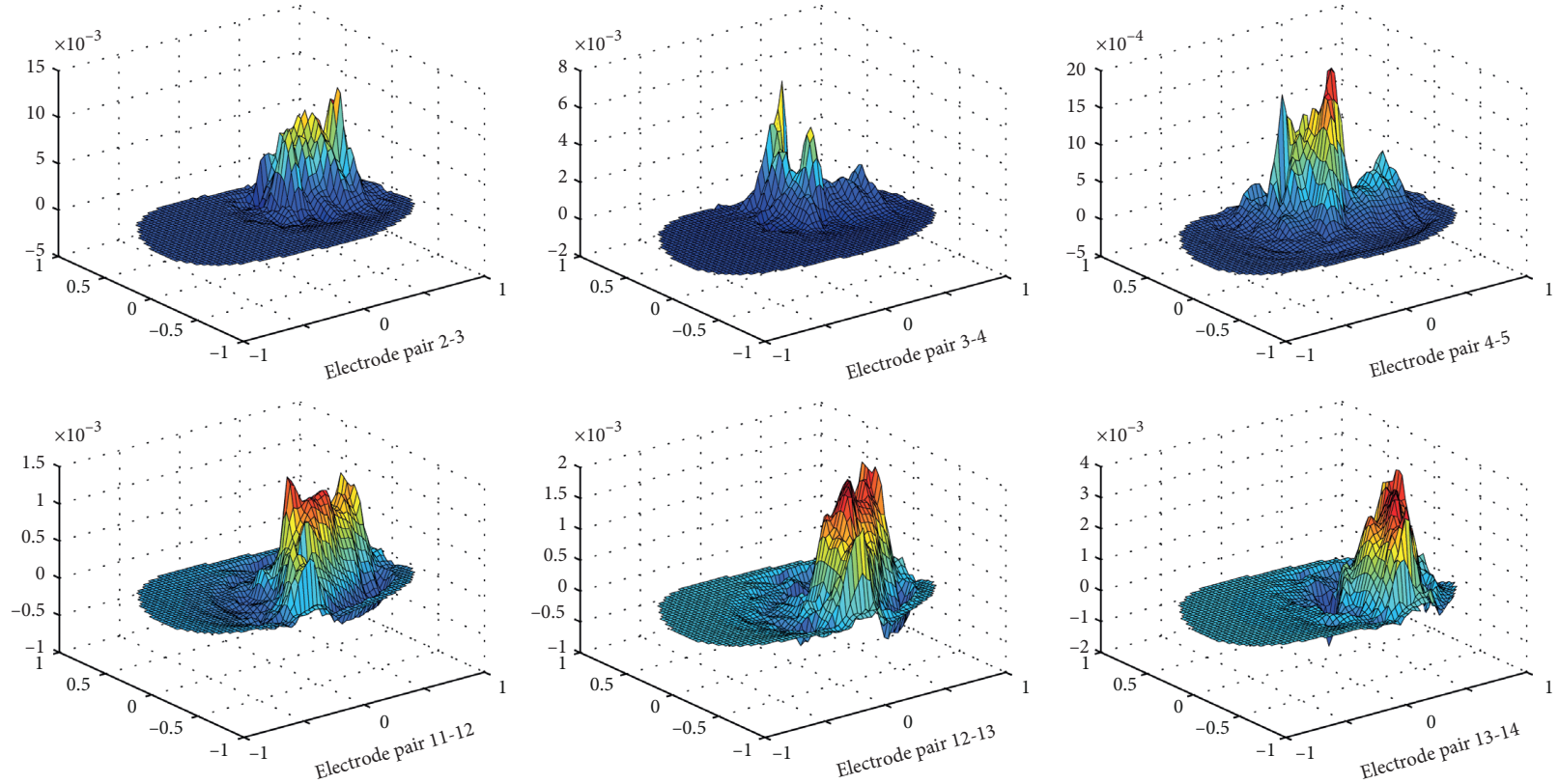

(c)
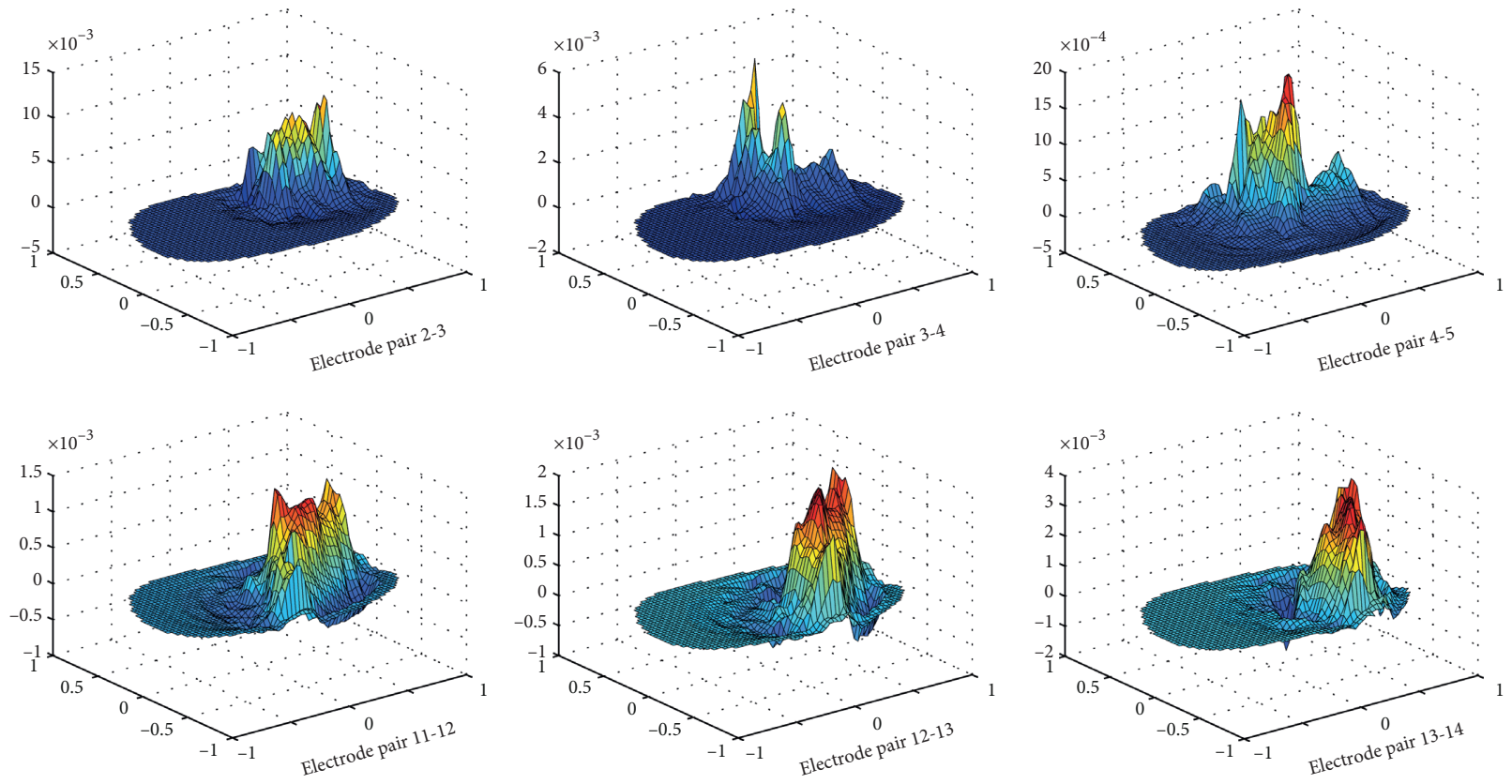

(d)

Figure 3: Continued. 

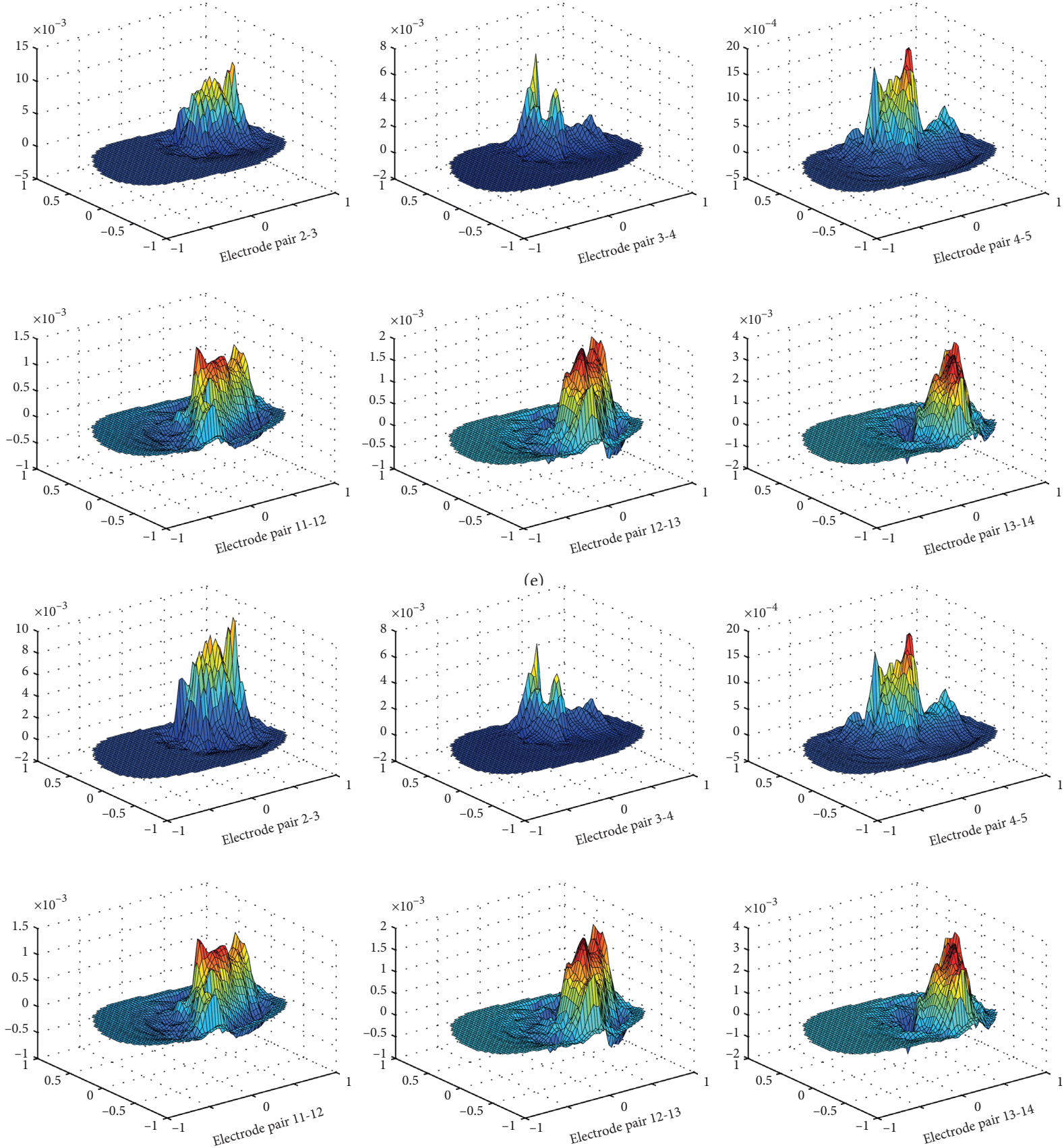

(f)

Figure 3: Continued. 

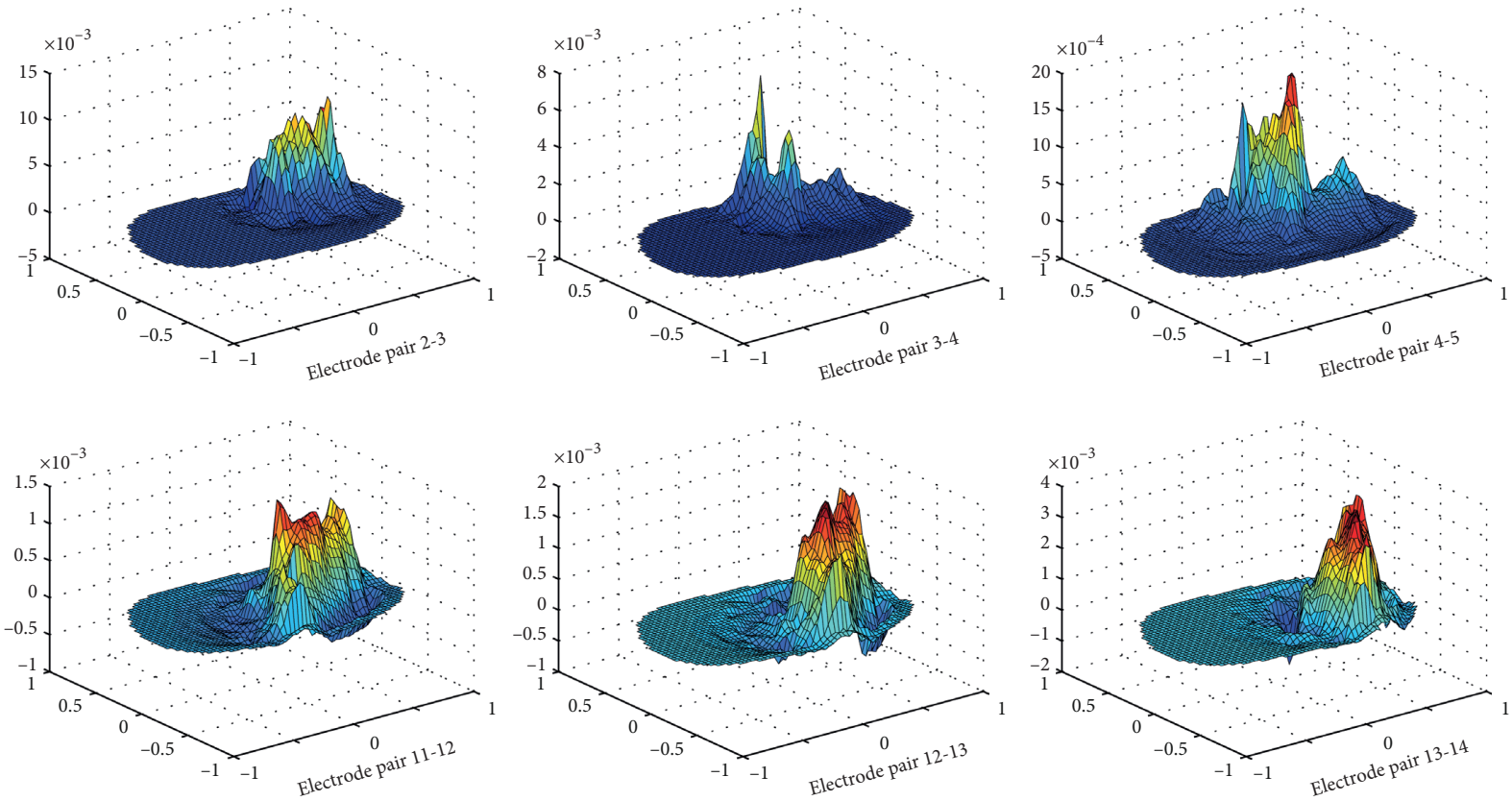

(g)

Figure 3: Schematic diagram of sensitivity distribution.

TABLE 1: Comparison of different parameters of the corresponding sensitivity matrices.

\begin{tabular}{lccccccc}
\hline Parameters & 1.8750 degrees & 3.7500 degrees & 5.6250 degrees & 7.5000 degrees & 9.3750 degrees & 11.2500 degrees & 13.1250 degrees \\
\hline Maximum & 0.0186 & 0.0192 & 0.0187 & 0.0192 & 0.0188 & 0.0189 & 0.0186 \\
Minimum & -0.0035 & -0.0029 & -0.0026 & -0.0025 & -0.0025 & -0.0025 & -0.0025 \\
$P$ & 6.3435 & 6.3314 & 6.3183 & 6.3003 & 6.2789 & 6.2507 & 6.2195 \\
\hline
\end{tabular}

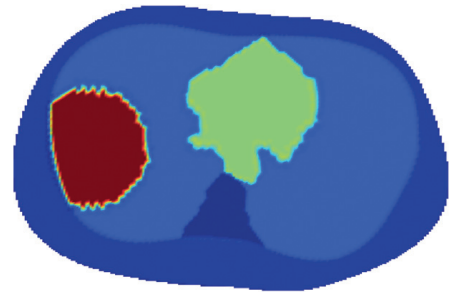

Distribution configuration 1

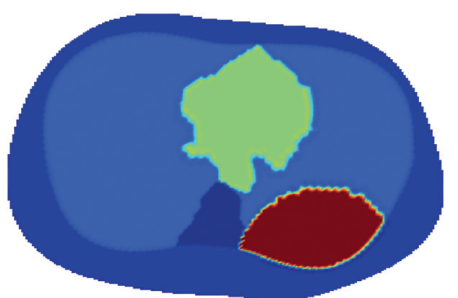

Distribution configuration 4

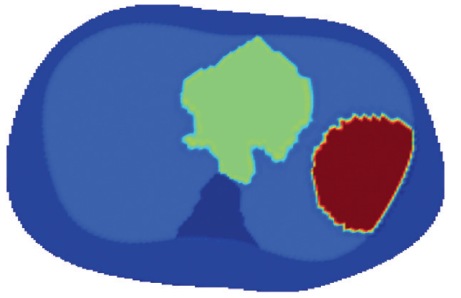

Distribution configuration 2

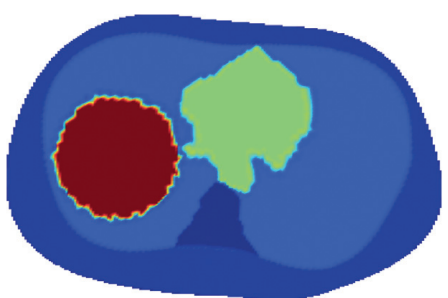

Distribution configuration 5

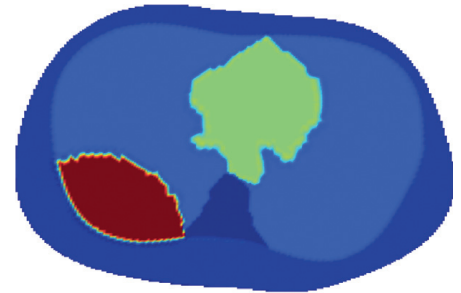

Distribution configuration 3

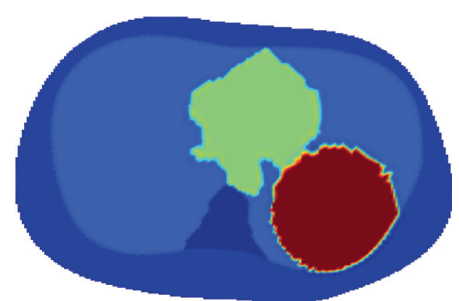

Distribution configuration 6

FIgURE 4: Configurations of the internal tissue and organ distribution of the FEM of the human lung. 


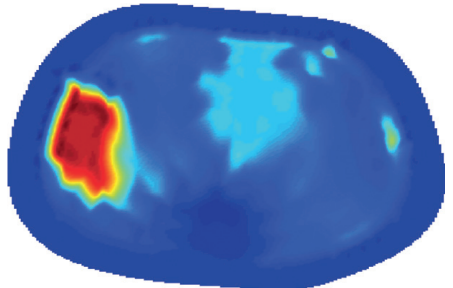

Distribution configuration 1

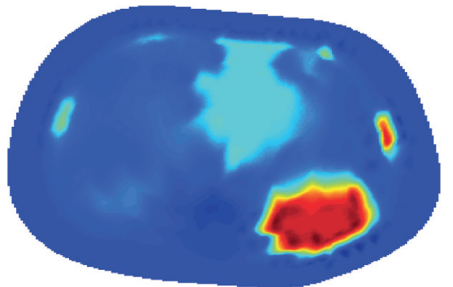

Distribution configuration 4

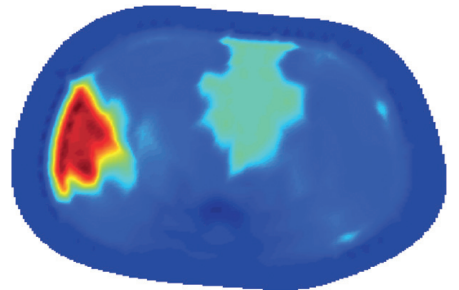

Distribution configuration 1

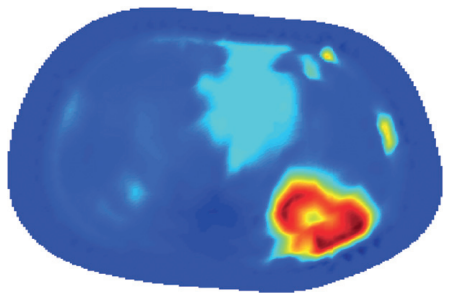

Distribution configuration 4

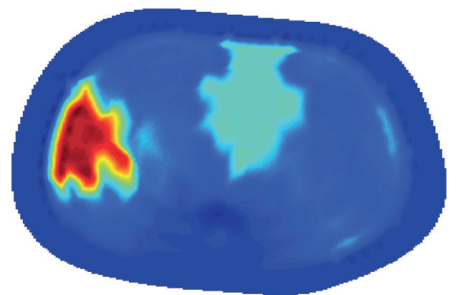

Distribution configuration 1

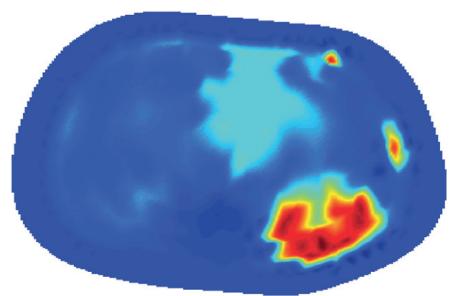

Distribution configuration 4

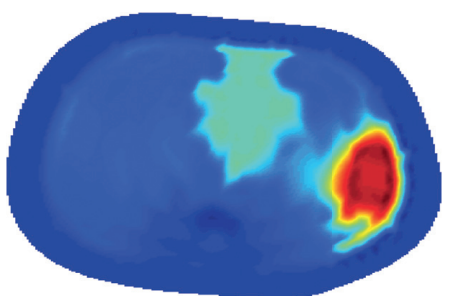

Distribution configuration 2

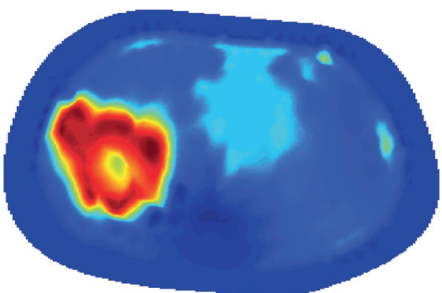

Distribution configuration 5

(a)

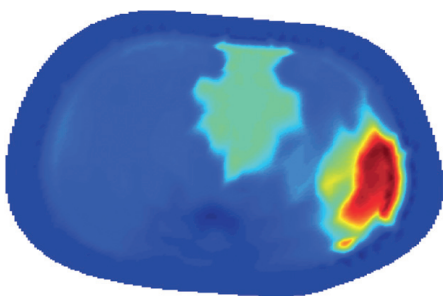

Distribution configuration 2

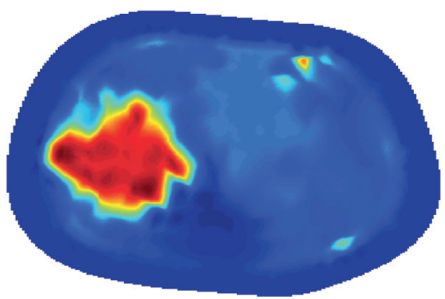

Distribution configuration 5

(b)

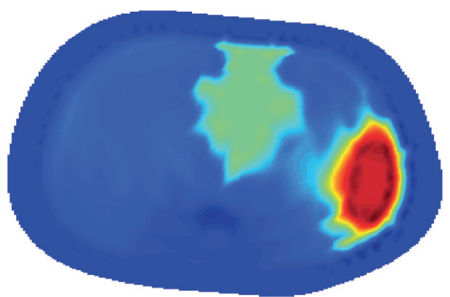

Distribution configuration 2

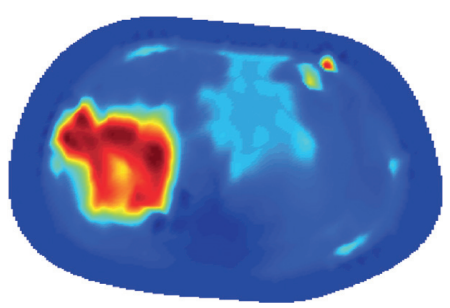

Distribution configuration 5

(c)

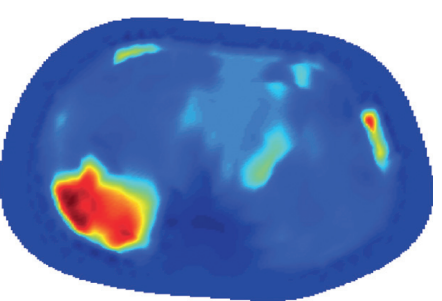

Distribution configuration 3

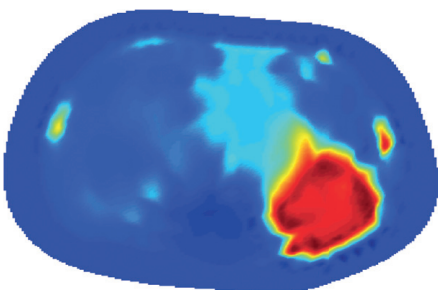

Distribution configuration 6

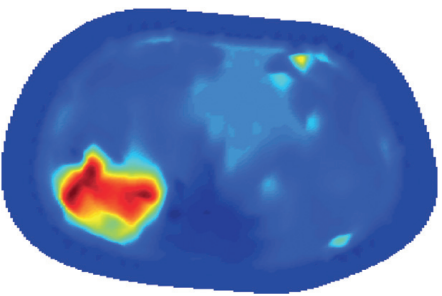

Distribution configuration 3

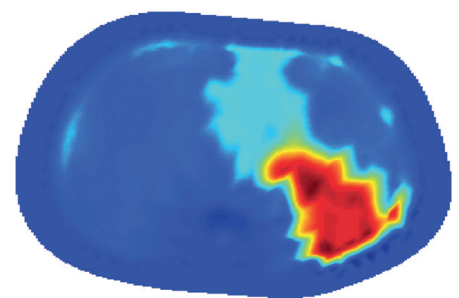

Distribution configuration 6

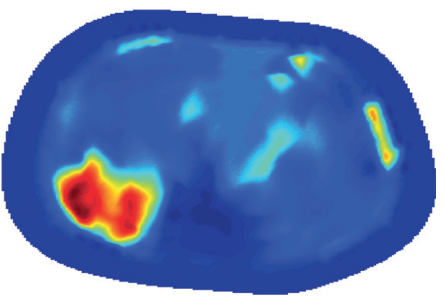

Distribution configuration 3

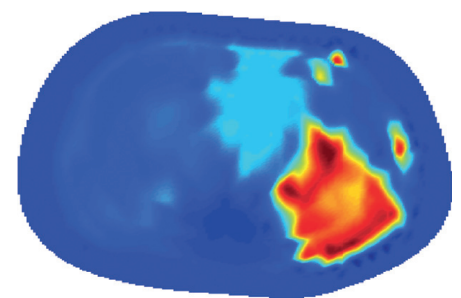

Distribution configuration 6

FIgURE 5: Continued. 


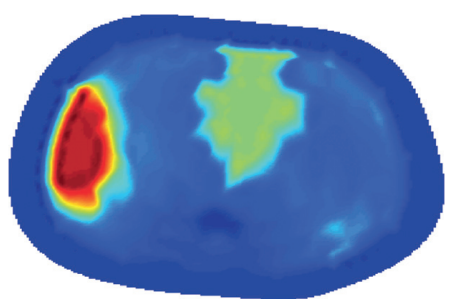

Distribution configuration 1

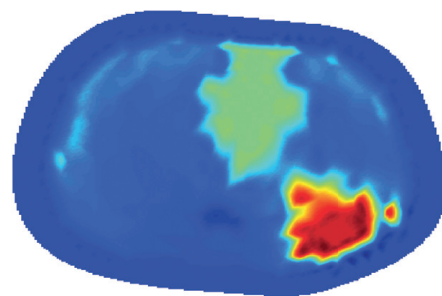

Distribution configuration 4

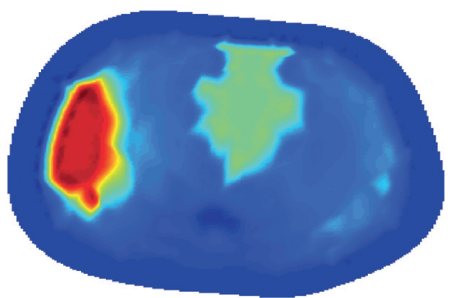

Distribution configuration 1

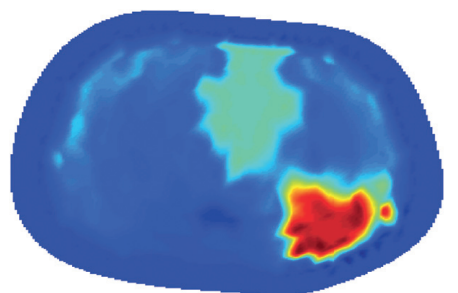

Distribution configuration 4

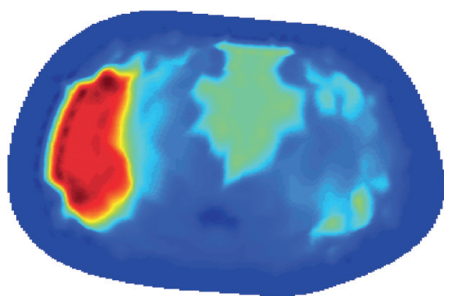

Distribution configuration 1

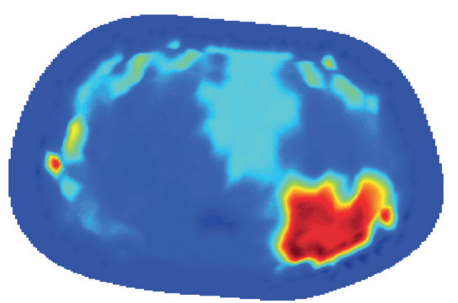

Distribution configuration 4

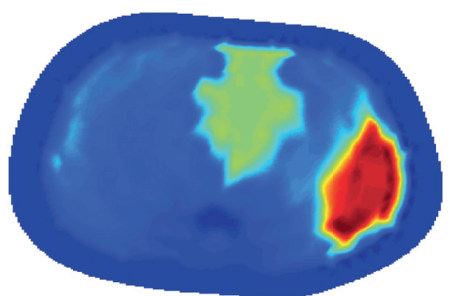

Distribution configuration 2

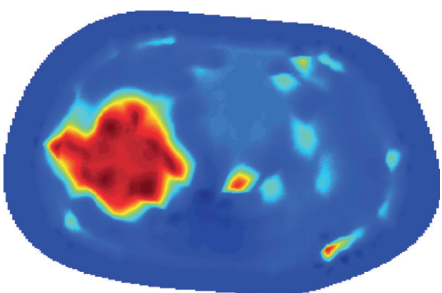

Distribution configuration 5

(d)

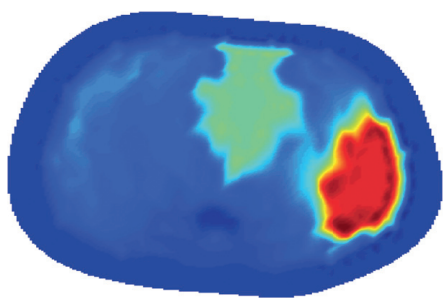

Distribution configuration 2

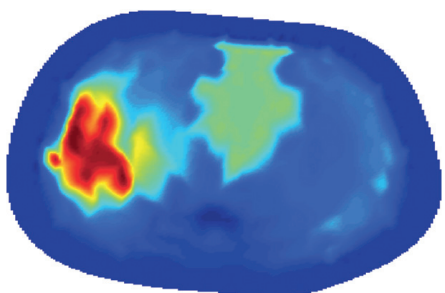

Distribution configuration 5

(e)

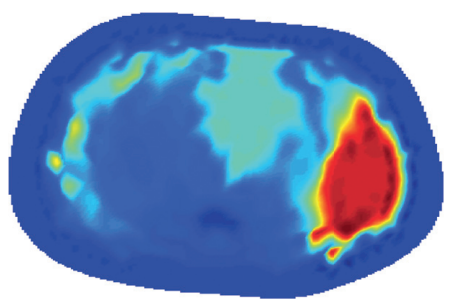

Distribution configuration 2

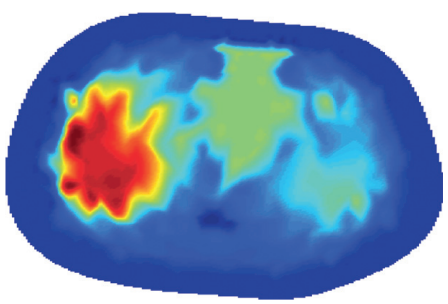

Distribution configuration 5

(f)

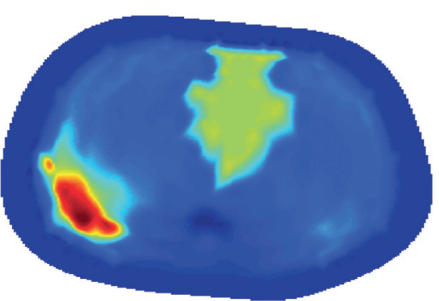

Distribution configuration 3

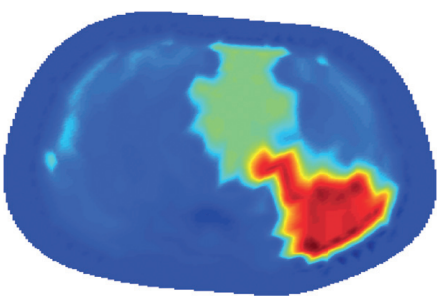

Distribution configuration 6

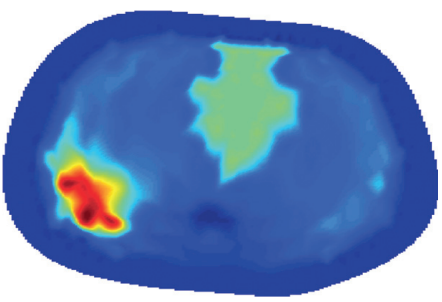

Distribution configuration 3

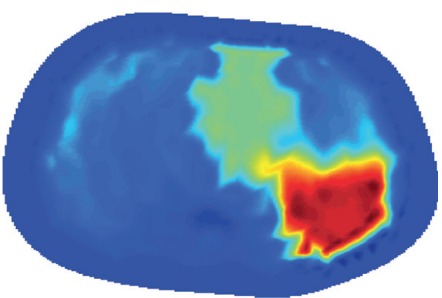

Distribution configuration 6

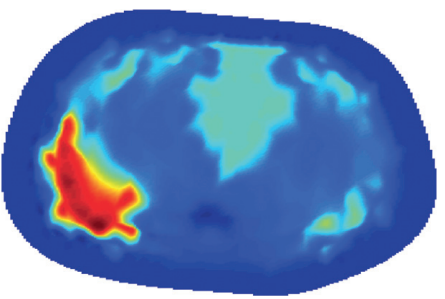

Distribution configuration 3

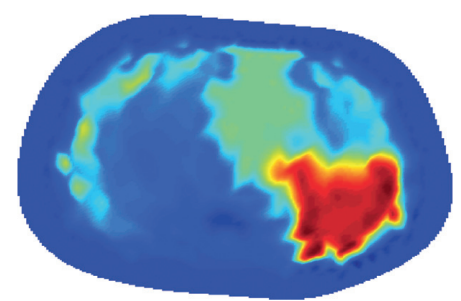

Distribution configuration 6

Figure 5: Continued. 


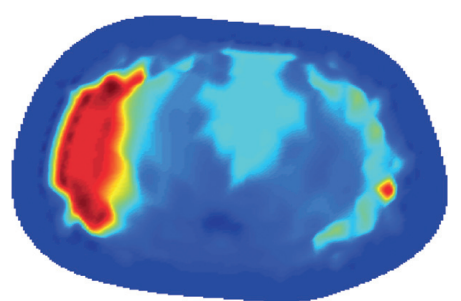

Distribution configuration 1

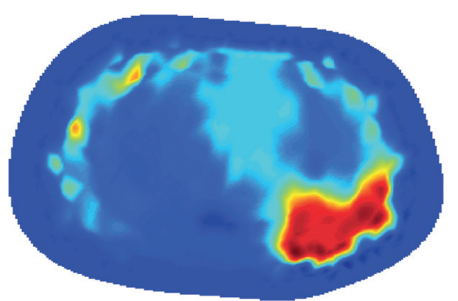

Distribution configuration 4

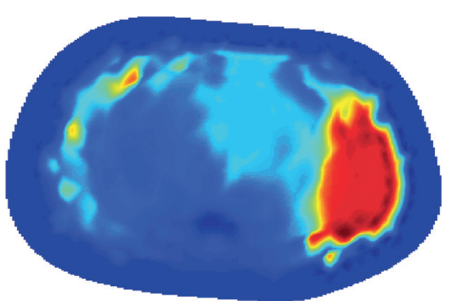

Distribution configuration 2

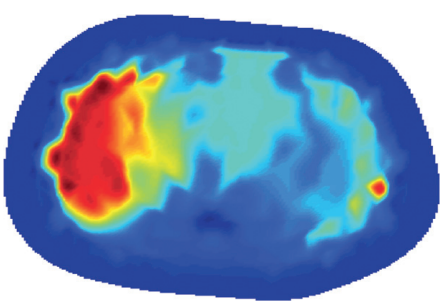

Distribution configuration 5

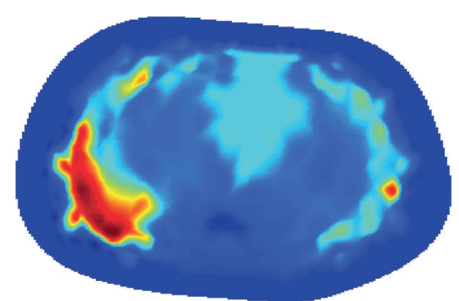

Distribution configuration 3

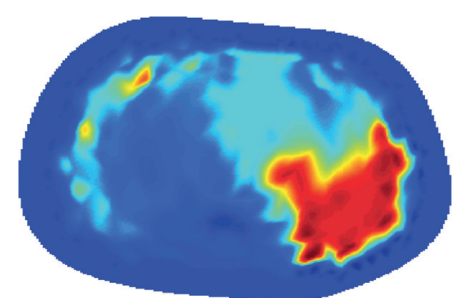

Distribution configuration 6

(g)

Figure 5: Reconstructed images. (a) The electrode angle $\theta$ is 1.8750 degrees. (b) The electrode angle $\theta$ is 3.7500 degrees. (c) The electrode angle $\theta$ is 5.6250 degrees. (d) The electrode angle $\theta$ is 7.5000 degrees. (e) The electrode angle $\theta$ is 9.3750 degrees. (f) The electrode angle $\theta$ is 11.2500 degrees. (g) The electrode angle $\theta$ is $13.1250^{\circ}$.

TABLE 2: Comparison of image correlation coefficients.

\begin{tabular}{lccccccc}
\hline $\begin{array}{l}\text { Distribution } \\
\text { Configuration }\end{array}$ & $\begin{array}{c}1.8750 \\
\text { degrees }\end{array}$ & $\begin{array}{c}3.7500 \\
\text { degrees }\end{array}$ & $\begin{array}{c}5.6250 \\
\text { degrees }\end{array}$ & $\begin{array}{c}7.5000 \\
\text { degrees }\end{array}$ & $\begin{array}{c}9.3750 \\
\text { degrees }\end{array}$ & $\begin{array}{c}11.2500 \\
\text { degrees }\end{array}$ & $\begin{array}{c}13.1250 \\
\text { degrees }\end{array}$ \\
\hline 1 & 0.8672 & 0.8374 & 0.8481 & 0.8450 & 0.8531 & 0.8260 & 0.7763 \\
2 & 0.8677 & 0.8531 & 0.8724 & 0.8604 & 0.8874 & 0.8250 \\
3 & 0.8248 & 0.8253 & 0.8034 & 0.7468 & 0.7615 & 0.7523 & 0.8009 \\
4 & 0.8544 & 0.8396 & 0.8481 & 0.8103 & 0.8102 & 0.7658 \\
5 & 0.9115 & 0.8804 & 0.8928 & 0.8491 & 0.8557 & 0.8736 \\
6 & 0.8890 & 0.8444 & 0.8754 & 0.8439 & 0.8646 & 0.8545 & 0.8180 \\
Average & 0.8691 & 0.8467 & 0.8567 & 0.8259 & 0.8388 & 0.8162 \\
value & & & & & & 0.7744 \\
\hline
\end{tabular}

TABLE 3: Comparison of image relative error (\%).

\begin{tabular}{llllllll}
\hline $\begin{array}{l}\text { Distribution } \\
\text { configuration }\end{array}$ & $\begin{array}{c}1.8750 \\
\text { degrees }\end{array}$ & $\begin{array}{c}3.7500 \\
\text { degrees }\end{array}$ & $\begin{array}{c}5.6250 \\
\text { degrees }\end{array}$ & $\begin{array}{c}7.5000 \\
\text { degrees }\end{array}$ & $\begin{array}{c}9.3750 \\
\text { degrees }\end{array}$ & $\begin{array}{c}11.2500 \\
\text { degrees }\end{array}$ & $\begin{array}{c}13.1250 \\
\text { degrees }\end{array}$ \\
\hline 1 & 42.3959 & 44.9342 & 44.2451 & 41.9743 & 40.5445 & 44.6119 \\
2 & 40.9719 & 42.8324 & 39.3069 & 39.6172 & 35.6991 & 44.3858 \\
3 & 46.4351 & 47.8764 & 48.9121 & 51.8914 & 50.8653 & 51.0591 & 47.8141 \\
4 & 42.5821 & 45.7429 & 43.8716 & 46.1569 & 46.4721 & 50.4642 \\
5 & 34.8015 & 39.3734 & 38.2171 & 43.1964 & 41.9310 & 37.2090 \\
6 & 37.9780 & 43.8795 & 40.8065 & 42.5448 & 39.7991 & 40.0577 & 45.0131 \\
Average & 40.8608 & 44.1065 & 42.5599 & 44.2302 & 42.5519 & 44.6313 & 45.1568 \\
value & & & & & & & 49.4449 \\
\hline
\end{tabular}

TABLE 4: Comparison of absolute errors.

\begin{tabular}{lccccccc}
\hline $\begin{array}{l}\text { Distribution } \\
\text { Configuration }\end{array}$ & $\begin{array}{c}1.8750 \\
\text { degrees }\end{array}$ & $\begin{array}{c}3.7500 \\
\text { degrees }\end{array}$ & $\begin{array}{c}5.6250 \\
\text { degrees }\end{array}$ & $\begin{array}{c}7.5000 \\
\text { degrees }\end{array}$ & $\begin{array}{c}9.3750 \\
\text { degrees }\end{array}$ & $\begin{array}{c}11.2500 \\
\text { degrees }\end{array}$ & $\begin{array}{c}13.1250 \\
\text { degrees }\end{array}$ \\
\hline 1 & 0.1129 & 0.0719 & 0.0591 & 0.1259 & 0.1398 & 0.1573 \\
2 & 0.0901 & 0.1018 & 0.0809 & 0.1386 & 0.1228 & 0.1185 \\
3 & 0.1558 & 0.1783 & 0.1739 & 0.2023 & 0.1897 & 0.1562 & 0.1645 \\
4 & 0.0809 & 0.1142 & 0.0860 & 0.1256 & 0.1065 & 0.1467 & 0.1650 \\
5 & 0.1219 & 0.1641 & 0.1214 & 0.2009 & 0.1582 & 0.1905 \\
6 & 0.1053 & 0.0776 & 0.0970 & 0.1070 & 0.1145 & 0.1413 & 0.1370 \\
Average & 0.1112 & 0.1180 & 0.1030 & 0.1500 & 0.1386 & 0.1518 \\
value & & & & & & 0.1551 \\
\hline
\end{tabular}




\section{Conclusion}

To improve the accuracy of solving the inverse problem for human lung ERT, first, the FEM for human lung ERT was set up based on prior knowledge. On this basis, the results were compared and analysed in the aspects of the morbidity degrees of sensitivity matrix and Hessian matrix, the uniformity of sensitivity distribution, and the quality of reconstructed images at electrode angles of 1.8750 degrees, 3.7500 degrees, 5.6250 degrees, 7.5000 degrees, 9.3750 degrees, 11.2500 degrees, and 13.1250 degrees, respectively, and the optimisation of electrode width was obtained. In the future, by adjusting the position of the outermost triangular finite element node, the number of array electrode widths can be increased. On this basis, the number of array electrodes, array electrode widths, and data acquisition mode can be comprehensively optimised.

\section{Data Availability}

The data used to support the findings of the study can be obtained from the author upon request.

\section{Conflicts of Interest}

The author declares no conflicts of interest.

\section{Acknowledgments}

This work was supported by the Huainan Normal University Research and Innovation Team: Intelligent Detection Technology Research and Innovation Team (XJTD202009) and Key Project of Excellent Young Talents Supporting Program of Colleges and Universities in Anhui Province in 2019 under Grant gxyqZD2019065.

\section{References}

[1] National Health and Family Planning Commission 2013, China Health and Family Planning Statistical Yearbook, China Union Medical University Press, Beijing, China, 2013.

[2] National Health and Family Planning Commission 2014, China Health and Family Planning Statistical Yearbook, China Union Medical University Press, Beijing, China, 2014.

[3] National Health and Family Planning Commission 2015, China Health and Family Planning Statistical Yearbook, China Union Medical University Press, Beijing, China, 2015.

[4] National Health and Family Planning Commission 2016, China Health and Family Planning Statistical Yearbook, China Union Medical University Press, Beijing, China, 2016.

[5] National Health and Family Planning Commission 2017, China Health and Family Planning Statistical Yearbook, China Union Medical University Press, Beijing, China, 2017.

[6] National Health Commission, China Health Statistical Yearbook 2018, China Union Medical University Press, Beijing, China, 2018.

[7] National Health Commission, China Health Statistical Yearbook 2019, China Union Medical University Press, Beijing, China, 2019.

[8] H. He, Yi Chi, L. Yun et al., "Influence of overdistension/ recruitment induced by high positive end-expiratory pressure on ventilation-perfusion matching assessed by electrical impedance tomography with saline bolus," Critical Care, vol. 24, no. 1, 2020.

[9] H. Garde, "Reconstruction of piecewise constant layered conductivities in electrical impedance tomography," Communications in Partial Differential Equationuations, vol. 45, no. 9, 2020.

[10] C. Secombe, A. D. Waldmann, G. Hosgood, and M. Mosing, "Evaluation of histamine-provoked changes in airflow using electrical impedance tomography in horses," Equationuine Veterinary Journal, vol. 52, no. 4, 2020.

[11] B. Alistair, A. Kirill, and A. Adler, "Beneficial techniques for spatio-temporal imaging in electrical impedance tomography," Physiological Measurement, vol. 41, no. 6, 2020.

[12] Z. Li, J. Zhang, D. Liu, and J. Du, "CT image-guided electrical impedance tomography for medical imaging," IEEE Transactions on Medical Imaging, vol. 39, no. 6, 2020.

[13] G. Ma, H. Zhiliang, X. Wu, and X. Wang, "An optimal electrical impedance tomography drive pattern for humancomputer interaction applications," IEEE Transactions on Biomedical Circuits and Systems, vol. 14, no. 3, 2020.

[14] I. S. Hussam, R. S. Jordan, S. D. Craig, A. H. John, and W. K. Brian, "Distribution of ventilation measured by electrical impedance tomography in critically ill children," $R e$ spiratory Care, vol. 65 , no. 5, 2020.

[15] B. Li, J. M. Wang, Q. Wang, X. Y. Li, and X. Duan, "A novel gas/liquid two-phase flow imaging method through electrical resistance tomography with DDELM-AE sparse dictionary," Sensor Review, vol. 40, no. 4, 2020.

[16] A. Paglianti, G. Marotta, and G. Montante, "Applicability of electrical resistance tomography to the analysis of fluid distribution in haemodialysis modules," The Canadian Journal of Chemical Engineering, vol. 98, no. 9, 2020.

[17] C. Jan and L. Michalcová, "Impact damage detection in CFRP composite via electrical resistance tomography by means of statistical processing," Journal of Nondestructive Evaluation, vol. 39, no. 2, 2020.

[18] L. Xiao, "Optimization of hessian matrix in modified newtonraphson algorithm for electrical resistance tomography," European Journal of Electrical Engineering, vol. 21, no. 5, 2019.

[19] G. Forte, A. Albano, J Mark, E. H. Stitt, and E. Brunazzi, F. Alberini, "Assessing blending of non-Newtonian fluids in static mixers by planar laser-induced fluorescence and electrical resistance tomography," Chemical Engineering \& Technology, vol. 42, no. 8, 2019.

[20] F. Mirshekari and L. Pakzad, "Mixing of oil in water through electrical resistance tomography and response surface methodology," Chemical Engineering \& Technology, vol. 42, no. 5, 2019.

[21] J. Lei, Q. B. Liu, and X. Y. Wang, "Computational imaging method with a learned plug-and-play prior for electrical capacitance tomography," Cognitive Computation, vol. 12, no. $1,2020$.

[22] Q. Y. Tu and H. G. Wang, "Investigation of the riser crosssectional aspect ratio effect on the flow dynamics in circulating fluidized beds by electrical capacitance tomography," Transactions of the Institute of Measurement and Control, vol. 42, no. 4, 2020.

[23] V. Mosorov, M. Zych, R. Hanus, D. Sankowski, and S. Ayoub, "Improvement of flow velocity measurement algorithms based on correlation function and twin Plane electrical capacitance tomography," Sensors, vol. 20, no. 1, 2020.

[24] Z. Almutairi, M. Fayez, Al-Alweet, Y. A. Alghamdi, O. A. Almisned, and O. Y. Alothman, "Investigating the characteristics of two-phase flow using electrical capacitance 
tomography (ECT) for three pipe," Orientations Processes, vol. 8 , no. $1,2020$.

[25] C. Oo Maung, D. Kawashima, P. N. Darma, and M. Takei, "Real-time controlling particle distribution in pneumatic conveyance by electrical capacitance tomography with airflow injection system (ECT-AIS)," Advanced Powder Technology, vol. 31 , no. $6,2020$.

[26] J. Lei, Q. B. Liu, and X. Y. Wang, "Three-operator splitting scheme with the reference image regularization for electrical capacitance tomography," Neural Computing and Applications, vol. 31, no. 9, 2019.

[27] A. Kowalska, R. Banasiak, A. Romanowski, and D. Sankowski, "3D-Printed multilayer sensor structure for electrical capacitance tomography," Sensors, vol. 19, no. 15, 2019.

[28] Y. Lv, X. He, E. Zhao et al., "Spectral specific standardized low-resolution brain electromagnetic tomography default mode connectivity network and plasticity alterations in left vertebral artery stent patients," International Journal of Distributed Sensor Networks, vol. 16, no. 3, 2020.

[29] Q. Wang, L. Kun, R. Zhang et al., "Sparse defects detection and 3D imaging base on electromagnetic tomography and total variation algorithm," The Review of Scientific Instruments, vol. 90, no. 12, 2019.

[30] C. Guo, Z. Yang, X. Wu, T. Tan, and K. Zhao, "Application of an adaptive multi-population parallel genetic algorithm with constraints in electromagnetic tomography with Incomplete projections," Applied Sciences, vol. 9, no. 13, 2019.

[31] J. Ye-Hwa, T.-H. Eom, Y.-H. Kim, S.-Y. Chung, L. In-Goo, and J.-M. Kim, "Source localization of epileptiform discharges in childhood absence epilepsy using a distributed source model: a standardized, low-resolution, brain electromagnetic tomography (sLORETA) study," Neurological Sciences, vol. 40, no. 5, 2019.

[32] S. Prinsloo, D. I. Rosenthal, R. Lyle et al., "Exploratory study of low resolution electromagnetic tomography (LORETA) real-time Z-score feedback in the treatment of pain in patients with head and neck cancer," Brain Topography, vol. 32, no. 2, 2019.

[33] J. Ye-Hwa, E. Tae-Hoon, K. Young-Hoon, S.-Y. Chung, L. InGoo, and K. Jung-Min, "Changes in background electroencephalographic activity in benign childhood epilepsy with centrotemporal spikes after oxcarbazepine treatment: a standardized low-resolution brain electromagnetic tomography (sLORETA) study," BMC Neurology, vol. 19, no. 1, 2019.

[34] L. Xiao, Finite Element Model and Image Reconstruction Algorithm of Electrical Resistance Tomography, Science Press, Beijing, China, 2019.

[35] L. Xiao, H. Wang, and X. Xu, "Improved Newton-Raphson algorithm for electrical resistance tomography image reconstruction," Proceedings of the CSEE, vol. 32, no. 8, 2012. 